\title{
Diabetes Gestacional e o Impacto do Actual Rastreio
}

\author{
Gestational Diabetes and the New Screening Test's Impact
}

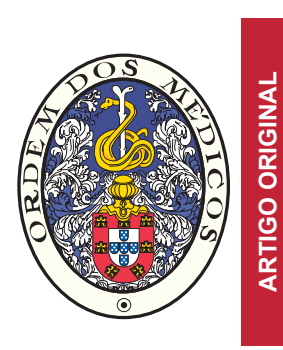

\author{
Ana Catarina MASSA ${ }^{1}$, Ricardo RANGEL ${ }^{2}$, Manuela CARDOSO ${ }^{3}$, Ana CAMPOS $^{1}$ \\ Acta Med Port 2015 Jan-Feb;28(1):29-34
}

\section{RESUMO}

Introdução: Em 2011, foi introduzido um novo rastreio para a diabetes gestacional que permitiu um diagnóstico mais precoce e de maior número de casos com o intuito de reduzir complicações maternas e perinatais. O objectivo deste estudo foi avaliar a prevalência da diabetes gestacional, comparar resultados obstétricos e perinatais do anterior e presente rastreio e os resultados e realização da prova de reclassificação pós-parto.

Material e Métodos: Estudo retrospectivo em gestações simples e diabetes gestacional diagnosticados em 2009 ( $n=223$ e 2012 $(n=237)$, vigiadas na Maternidade Dr. Alfredo da Costa, Portugal. Após consulta de processos clínicos procedeu-se à análise de características demográficas, história médica e obstétrica, aumento ponderal durante a gravidez, idade gestacional do diagnóstico, terapêutica utilizada, resultados perinatais e reclassificação pós-parto, seguida de comparação destas variáveis entre os anos de 2009 e 2012.

Resultados: Em 2012, houve maior prevalência de diabetes gestacional, ganho ponderal inferior $(p<0,001)$, maior recurso à terapêutica farmacológica $(p<0,001)$ e aumento dos casos diagnosticados no primeiro e segundo trimestres $(p<0,001)$. Relativamente aos resultados neonatais, o peso médio do recém-nascido ao nascer foi significativamente menor $(p=0,001)$ com diminuição dos recém-nascidos grandes para a idade gestacional $(p=0,002)$. A taxa de reclassificação pós-parto foi semelhante nos dois anos mas em 2012 houve um aumento dos resultados normais e diminuição das anomalias da glicémia em jejum.

Discussão: Critérios mais apertados do actual rastreio permitiram a redução da maioria das complicações da diabetes gestacional levantando novas questões.

Conclusão: A introdução do actual rastreio resultou num aumento de prevalência, diagnóstico mais precoce e redução da macrossomia.

Palavras-chave: Diabetes Gestacional; Rastreio.

\section{ABSTRACT}

Introduction: In 2011, a new screening test for gestational diabetes was introduced which allowed an earlier and larger diagnosis with the goal of reducing maternal and perinatal complications. The aim of our study was to evaluate the prevalence of gestational diabetes, compare maternal and perinatal outcomes with the previous and present screening tests and analyze postpartum screening results. Material and Methods: Retrospective study of women with singletons and gestational diabetes diagnosed during 2009 ( $n=223$ ) and 2012 ( $n=237)$, at Maternidade Dr. Alfredo da Costa, Portugal. Analysis of clinical charts and assessment of demographic data, medical and obstetric history, weight gain during pregnancy, gestational age at diagnosis, treatment regimens, neonatal outcomes and postpartum screening results, followed by comparison of these variables between the years of 2009 and 2012 .

Results: In 2012, there was an increased gestational diabetes prevalence, lower weight gain during pregnancy $(p<0.001)$, more frequent use of pharmacological therapy $(p<0.001)$ and more diagnosed cases during first and second trimester $(p<0.001)$. As for neonatal outcomes, in this group, the medium weight at birth was significantly lower $(p=0.001)$ with a decrease of newborns great for gestational age $(p=0.002)$. Postpartum screening rate was similar among both groups but in 2012 there was an increase of normal results and a decrease of impaired fasting glucose.

Discussion: Tighter criteria of the current screening test resulted in reduction of the majority of gestational diabetes complications but raised new questions.

Conclusion: The introduction of the current screening test resulted in an increased prevalence, earlier diagnosis and reduction of macrosomia.

Keywords: Gestational Diabetes; Mass Screening.

\section{INTRODUÇÃO}

A diabetes gestacional (DG) é uma das complicações médicas mais frequentes da gravidez, cuja incidência tem vindo a crescer nos últimos anos. Definida como uma intolerância aos hidratos de carbono de intensidade variável que é reconhecida pela primeira vez ou diagnosticada durante a gravidez, apresenta uma prevalência estimada de 5 a $7 \%$ e habitualmente resolve após o parto. ${ }^{1-7}$

Diversos factores de risco foram identificados, como idade materna avançada, etnia (Hispânica, Afro-Americana e Asiática), índice de massa corporal (IMC) pré-concepcional elevado, antecedentes de DG ou antecedentes familiares de diabetes mellitus tipo 1 ou tipo 2.5,6,8

Estudos anteriores mostraram uma relação entre a DG e desfechos adversos a curto e longo prazo. As doenças hipertensivas da gravidez, como a hipertensão induzida pela gravidez (HIG) e pré-eclâmpsia (PE), e o risco de de-

1. Serviço de Medicina Materno-Fetal. Maternidade Alfredo da Costa. Centro Hospitalar Lisboa Central. Lisboa. Portugal.

2. Serviço de Endocrinologia. Hospital Curry Cabral. Centro Hospitalar Lisboa Central. Lisboa. Portugal.

3. Serviço de Nutrição. Maternidade Alfredo da Costa. Centro Hospitalar Lisboa Central. Lisboa. Portugal.

Recebido: 18 de Março de 2014 - Aceite: 07 de Julho de 2014 | Copyright @ Ordem dos Médicos 2015 
senvolver diabetes mellitus tipo 2 no futuro, em média 22 a 28 anos depois, são exemplos de complicações maternas. Desfechos fetais como Recém-Nascidos (RN) Grandes para a Idade Gestacional (GIG) / fetos macrossómicos, aumento da taxa de cesarianas, de distócia de ombros, de síndrome de dificuldade respiratória do $\mathrm{RN}$ e de complicações metabólicas podem estar presentes nesta patologia. ${ }^{1,4-6}$

De 2000 a 2010 em Portugal, o diagnóstico de DG preconizado baseava-se numa abordagem em dois passos inicialmente era realizada uma prova de rastreio com uma sobrecarga de $50 \mathrm{~g}$ de glucose oral no $2^{\circ}$ e $3^{\circ}$ trimestres, ou no primeiro trimestre se houvesse factores de risco, seguida de uma prova diagnóstica com uma sobrecarga de 100 $\mathrm{g}$ de glucose oral após um teste de rastreio positivo. ${ }^{8}$

O estudo HAPO (Hyperglycemia and Adverse Pregnant Outcomes) veio revolucionar o rastreio desta doença. Trata-se de um estudo multicêntrico que avaliou a tolerância à glicose entre as 24 e as 32 semanas de gestação - utilizando para o diagnóstico de DG critérios de glicémia em jejum no primeiro trimestre $\geq 92 \mathrm{mg} / \mathrm{dL}$ ou um valor alterado na prova de tolerância à glucose oral (PTGO $75 \mathrm{~g}-0 \mathrm{~h} \geq 92$ $\mathrm{mg} / \mathrm{dL}, 1 \mathrm{~h} \geq 180 \mathrm{mg} / \mathrm{dl}, 2 \mathrm{~h} \geq 153 \mathrm{mg} / \mathrm{dl}$ ) - e que demonstrou uma relação linear entre os níveis de glicémia materna e as morbilidades materna, fetal e perinatal. ${ }^{2}$ Este rastreio foi implementado em Portugal em 2011 e o período entre as 24 e as 28 semanas foi recomendado como o ideal para a sua realização, uma vez que abrangeria um maior número de casos diagnosticados. Foi apoiado pela Direcção Geral de Saúde, Sociedade Portuguesa de Endocrinologia, Diabetes e Metabolismo, Sociedade Portuguesa de Diabetologia, Sociedade de Obstetrícia e Medicina Materno-Fetal e Secção de Neonatologia da Sociedade Portuguesa de Pediatria e teve como objectivo o diagnóstico precoce e de um maior número de grávidas, redução de complicações hipertensivas, macrossomia, cesarianas e distócia de ombros através da monitorização glicémia apertada e instituição terapêutica precoce. ${ }^{1-4,7}$ Este rastreio foi também adoptado por algumas Sociedades Internacionais nomeadamente pela International Association of Diabetes and Pregnancy Study Groups (IADPSG) e pela American Diabetes Association. $^{3}$

Após o parto está recomendado a todas as mulheres com este diagnóstico a realização da prova de reclassificação - em 6-8 semanas - através de uma PTGO com sobrecarga de $75 \mathrm{~g}$ de glucose com duas determinações: às $0 \mathrm{~h}$ (jejum) e às $2 \mathrm{~h} \cdot{ }^{6,7,8} \mathrm{~A}$ prova é considerada negativa se o primeiro valor for inferior a $110 \mathrm{mg} / \mathrm{dL}$ e se o segundo for inferior a $140 \mathrm{mg} / \mathrm{dL}$. Os critérios para determinar se uma prova de reclassificação pós-parto é considerada como normal, anomalia da glicémia em jejum, tolerância diminuída à glicose ou com o diagnóstico de diabetes mellitus tipo 2 encontram-se definidos na tabela $1 .{ }^{7}$

O objectivo do estudo foi comparar os resultados obstétricos e perinatais de grávidas submetidas ao anterior e ao presente rastreio e avaliar os resultados da prova de reclassificação pós-parto (2009 vs. 2012). Foi também avaliada a prevalência de DG.

A escolha dos anos de 2009 e 2012 teve como objectivo permitir a comparação dos resultados obstétricos e perinatais entre os dois métodos de rastreio: o primeiro, em 2009, que se baseava numa prova de rastreio e que exigia a prova diagnóstica para confirmação e o segundo, em 2012, com o actual rastreio, que requer apenas um valor alterado no teste de rastreio para se efectuar o diagnóstico.

\section{MATERIAL E MÉTODOS}

Foi realizado um estudo retrospectivo na Maternidade Alfredo da Costa - centro perinatal terciário. Trata-se de um estudo de grávidas com gestações únicas seguidas na nossa Consulta de Diabetes com o diagnóstico de DG nos anos de 2009 e 2012. Os autores avaliaram os seguintes parâmetros:

- Características demográficas: idade materna e IMC pré-concepcional;

- Antecedentes obstétricos: paridade, antecedentes de diabetes gestacional e de macrossomias;

- Antecedentes familiares em primeiro grau de diabetes mellitus tipo 1 ou tipo 2;

- Trimestre de diagnóstico de DG, terapêutica utilizada (nutricional e/ou farmacológica - insulina, metformina ou ambos) e valor de $\mathrm{HbA} 1 \mathrm{c}$ no terceiro trimestre;

- Aumento ponderal e complicações hipertensivas durante a gravidez;

- Variáveis do parto e recém-nascido (RN): idade gestacional (IG) no parto, tipo de parto (eutócico, distócico vaginal e cesariana), peso do RN ao nascer, Índice de Apgar (IA) do RN, percentil do RN ao nascer de acordo com a IG e sexo, distócia de ombros;

- Prova de reclassificação pós-parto.

Tabela 1 - Valores de referência para a prova de reclassificação

\begin{tabular}{lccc}
\hline Classificação & Jejum & & 2h após sobrecarga \\
\hline Normal & $<110 \mathrm{mg} / \mathrm{dL}$ & $\mathrm{E}$ & $<140 \mathrm{mg} / \mathrm{dL}$ \\
Anomalia da glicémia em jejum & $\geq 110 \mathrm{mg} / \mathrm{dL} e<126 \mathrm{mg} / \mathrm{dL}$ & $\mathrm{E}$ & $<140 \mathrm{mg} / \mathrm{dL}$ \\
Tolerância diminuída à glicose & $<126 \mathrm{mg} / \mathrm{dL}$ & $\mathrm{E}$ & $\geq 140 \mathrm{mg} / \mathrm{dL} e<200 \mathrm{mg} / \mathrm{dL}$ \\
Diabetes mellitus tipo 2 & $\geq 126 \mathrm{mg} / \mathrm{dL}$ & $\mathrm{OU}$ & $\geq 200 \mathrm{mg} / \mathrm{dL}$ \\
\hline
\end{tabular}


Procedeu-se à recolha de dados através da consulta de processos clínicos. Foram excluídas gestações gemelares ou com resultados perinatais desconhecidos.

A análise estatística foi efectuada recorrendo ao SPSS $20.0^{\circledR}$, através do recurso ao teste de $X^{2}$, teste exacto de Fischer e teste de Mann-Whitney. Foi utilizado um nível de significância estatística de $p \leq 0,05$.

\section{RESULTADOS}

Em 2009 foram seguidas na nossa Consulta de Diabetes 223 grávidas e em 2012, um total de 237 grávidas. Na Maternidade Dr. Alfredo da Costa efectuaram-se 5101 partos de gestações únicas em 2009 e 4412 partos em 2012, o que corresponde a uma prevalência de DG de $4,37 \%$ e $5,37 \%$, respectivamente, traduzindo-se num aumento de $22,8 \%$.

Em relação às características demográficas, antecedentes obstétricos e familiares os dois grupos eram semeIhantes entre si (Tabela 2).
Contudo, em 2012, verificou-se um aumento ponderal inferior $(11,01 \pm 5,35$ vs. 8,97 $\pm 6,44, p<0,001)$ e um aumento de casos diagnosticados no $1^{\circ}$ e $2^{\circ}$ trimestres (T) $\left(1^{\circ} \mathrm{T}-1,8 \%, 2^{\circ} \mathrm{T}-26,5 \%, 3^{\circ} \mathrm{T}-71,7 \%\right.$ vs. $1^{\circ} \mathrm{T}-30,8 \%$, $\left.2^{\circ} \mathrm{T}-53,2 \%, 3^{\circ} \mathrm{T}-16 \%, p<0,001\right)$. Registou-se ainda um maior recurso à terapêutica farmacológica associada à terapêutica nutricional em detrimento do uso exclusivo desta última $(16,1 \%$ vs. 38,8\%, $p<0,001)$ (Tabela 3). Relativamente à terapêutica farmacológica, no ano de 2009 apenas foi usada a insulina (36 casos / 16\%) e em 2012, a insulina (57 casos / 24\%), a metformina (23 casos / 10\%) ou a associação de ambos os fármacos (12 casos / 5\%) foram as utilizadas. Contudo, nos dois rastreios a terapêutica nutricional exclusiva permaneceu como a mais frequente (187 casos / 84\% em 2009 vs. 145 casos / 61\% em 2012).

Não houve diferenças com significado estatístico relativamente à média da $\mathrm{HbA} 1 \mathrm{c}$ do $3^{\circ}$ trimestre $(5,39 \pm 1,0$ vs. $5,58 \pm 0,5, p>0,05)$ ou à existência de complicações hipertensivas (Tabela 3 ).

Tabela 2 - Características demográficas e antecedentes obstétricos e familiares no ano de 2009 e 2012

\begin{tabular}{|c|c|c|c|}
\hline & $\begin{array}{c}2009 \\
(n=223)\end{array}$ & $\begin{array}{c}2012 \\
(n=237)\end{array}$ & $p$ \\
\hline Idade materna (anos) & $32,7 \pm 4,97$ & $33 \pm 5,44$ & NS \\
\hline $\begin{array}{l}\text { Paridade } \\
\text { Nulípara } \\
\text { Não-nulípara }\end{array}$ & $\begin{array}{c}96(43,1 \%) \\
127(56,9 \%)\end{array}$ & $\begin{array}{l}114(48,1 \%) \\
123(51,9 \%)\end{array}$ & NS \\
\hline IMC pré-concepcional $\left(\mathrm{kg} / \mathrm{m}^{2}\right)$ & $25,9 \pm 6,69$ & $26,8 \pm 6,82$ & NS \\
\hline Diabetes em gravidez anterior & $25(19,7 \%)$ & $27(22 \%)$ & NS \\
\hline Macrossomia fetal & $13(10,2 \%)$ & $12(9,8 \%)$ & NS \\
\hline História familiar de Diabetes & $130(58,3 \%)$ & $153(64,5 \%)$ & NS \\
\hline
\end{tabular}

NS - não significativo, IMC - índice de massa corporal.

Tabela 3 - Variáveis da gravidez no ano de 2009 e 2012

\begin{tabular}{|c|c|c|c|}
\hline & $\begin{array}{c}2009 \\
(n=223)\end{array}$ & $\begin{array}{c}2012 \\
(n=237)\end{array}$ & $p$ \\
\hline \multicolumn{4}{|l|}{ IG do diagnóstico por trimestre } \\
\hline $1^{\circ} \mathrm{T}$ & $4(1,8 \%)$ & $73(30,8 \%)$ & \multirow{3}{*}{$<0,001$} \\
\hline $2^{\circ} \mathrm{T}$ & $59(26,5 \%)$ & $126(53,2 \%)$ & \\
\hline $3^{\circ} \mathrm{T}$ & $160(71,7 \%)$ & $38(16 \%)$ & \\
\hline Aumento ponderal (kg) & $11,01 \pm 5,35$ & $8,97 \pm 6,44$ & $<0,001$ \\
\hline $\mathrm{HbA} 1 \mathrm{c}$ no $3^{\circ}$ trimestre & $5,39 \pm 1,0$ & $5,58 \pm 0,5$ & NS \\
\hline \multicolumn{4}{|l|}{ Terapêutica utilizada } \\
\hline Nutricional & $187(84 \%)$ & $145(61 \%)$ & \multirow[t]{2}{*}{$<0,001$} \\
\hline Farmacológica & $36(16 \%)$ & $92(39 \%)$ & \\
\hline Complicações hipertensivas & $20(9 \%)$ & $28(12 \%)$ & NS \\
\hline
\end{tabular}

IG - Idade Gestacional, T - trimestre, NS - não significativo. 
Tabela 4 - Variáveis do parto e do Recém-Nascido no ano de 2009 e 2012

\begin{tabular}{|c|c|c|c|}
\hline & $\begin{array}{c}2009 \\
(n=223)\end{array}$ & $\begin{array}{c}2012 \\
(n=237)\end{array}$ & $p$ \\
\hline IG do parto (semanas) & $38,4 \pm 1,42$ & $38,2 \pm 2,08$ & NS \\
\hline \multicolumn{4}{|l|}{ Tipo de parto } \\
\hline Eutócico & $103(46,2 \%)$ & $104(43,9 \%)$ & \\
\hline Distócico vaginal & $29(13 \%)$ & $45(19 \%)$ & \\
\hline Cesariana & $91(40,8 \%)$ & $88(37,1 \%)$ & NS \\
\hline Electiva por EP $>4,0 \mathrm{~kg}$ & $25 \%$ & $19,4 \%$ & \\
\hline Urgente por EFNT & $50 \%$ & $61,9 \%$ & \\
\hline Urgente por distócia & $46,2 \%$ & $28,6 \%$ & \\
\hline Distócia de ombros & $1(0,45 \%)$ & $3(1,27 \%)$ & NS \\
\hline Peso RN ao nascer (g) & $3339,1 \pm 524$ & $3163,1 \pm 582$ & 0,001 \\
\hline
\end{tabular}

Quanto aos resultados dos RN, a média de IG no parto, tipo de parto e distócia de ombros foram semelhantes entre os dois grupos. No entanto, tendencialmente registou-se uma redução do número de cesarianas com o actual rastreio. De facto, a taxa de cesarianas electivas por estimativa ponderal superior a $4000 \mathrm{~g}$ e de cesarianas urgentes por distócia diminuiu. Por outro lado, verificou-se um aumento de cesarianas urgentes por estado fetal não tranquilizador (EFNT) (Tabela 4).

Relativamente ao peso do $\mathrm{RN}$ ao nascer, no ano de 2012 verificou-se que este foi significativamente menor $(3339,1 \pm 524,5$ vs. $3163,1 \pm 582,58, p=0,001)$. Houve diferença na comparação de RN adequado para a IG (AIG), leve para a IG (LIG) e GIG nos anos de 2009 e 2012 ( $p=$ $0,001)$. Verificou-se um aumento da taxa de RN AIG $(83 \%$ vs. $88 \%)$ e de RN LIG (6\% vs. $8 \%$ ) e diminuição da taxa de RN GIG (11\% vs. $4 \%)$. Contudo, apenas esta última teve significado estatístico $(p=0,002)$ (Tabela 5). São necessários mais estudos, com maior número de casos, para confirmar esta tendência.

A avaliação da relação entre a terapêutica utilizada e o percentil de peso do RN para a IG, permite afirmar que em 2009 o recurso à insulinoterapia associou-se a uma redução dos RN GIG quando comparados com a terapêutica nutricional (insulina - oito RN GIG, nutricional - 17 RN GIG, $p=0,038$ ) e, em 2012, a utilização de antidiabéticos orais

Tabela 5 - Percentil de peso do Recém-Nascido para a Idade Gestacional de acordo com o sexo no ano de 2009 e 2012

\begin{tabular}{|c|c|c|c|}
\hline & $\begin{array}{c}2009 \\
(n=223)\end{array}$ & $\begin{array}{c}2012 \\
(n=237)\end{array}$ & $p$ \\
\hline RN LIG ( $\leq$ P10) & $12(6 \%)$ & $18(8 \%)$ & NS \\
\hline RN AIG & $186(83 \%)$ & $210(88 \%)$ & NS \\
\hline RN GIG ( $\geq$ P90) & $25(11 \%)$ & $9(4 \%)$ & 0,002 \\
\hline
\end{tabular}

mostrou o mesmo resultado (metformina - três RN GIG, restantes terapêuticas, incluindo a nutricional, seis RN GIG, $p=0,046$ ). Contudo, em alguns dos grupos terapêuticos a amostra é de reduzida dimensão.

Ao considerar apenas os casos de cesariana urgente por EFNT verificamos que nas duas amostras a IG do parto e o peso do RN ao nascer são semelhantes $(37,4 \pm 4,32$ vs. $37,8 \pm 2,35, p>0,05 ; 2955 \mathrm{~g} \pm 982,5$ vs. $3107 \pm 721,2, p>$ $0,05)$.

No ano de $2009,79,8 \%$ das mulheres com DG fizeram a prova de reclassificação pós-parto. Destas, $86 \%(n=153)$ tiveram uma prova normal, 5,6\% $(n=10)$ uma anomalia da glicémia em jejum, 6,7\% ( $n=12)$ uma tolerância diminuída à glicose oral e 1,7\% $(n=3)$ o diagnóstico de diabetes mellitus tipo 2. No ano de 2012, a taxa de reclassificação foi semelhante $-81 \%$. A prova foi normal em $94,3 \%(n=$ $181)$, detectou-se uma anomalia da glicémia em jejum em $0,5 \%(n=1)$, uma tolerância diminuída à glicose em $4,7 \%$ $(n=9)$ e houve um caso $(0,5 \%)$ de diabetes mellitus tipo 2 . A comparação dos dois métodos de rastreio mostrou um aumento de resultados normais na prova de reclassificação pós-parto $(p=0,007)$ e uma diminuição das anomalias da glicémia em jejum no ano de $2012(p=0,004)$ (Tabela 6$)$.

\section{DISCUSSÃO}

Com a progressiva alteração dos hábitos e estilos de vida nas últimas décadas, a nossa população tornou-se cada vez mais sedentária e com excesso de peso ou mesmo obesidade. $O$ crescente fluxo migratório de pessoas de países sul-americanos e asiáticos para o nosso País traduziu-se numa maior diversificação populacional. As dificuldades económicas e as exigências laborais levaram a que o projecto de maternidade seja, hoje em dia, colocado em segundo plano e adiado em grande parte das mulheres, tornando as grávidas actualmente mais velhas, com IMC pré-concepcional mais elevado e com maior risco de complicações da gravidez. Na nossa amostra, os autores concluíram que as características maternas foram semeIhantes nos dois anos. 
Tabela 6 - Reclassificação pós-parto no ano de 2009 e 2012

\begin{tabular}{|c|c|c|c|}
\hline & $\begin{array}{c}2009 \\
(n=178 / 79,8 \%)\end{array}$ & $\begin{array}{c}2012 \\
(n=192 / 81 \%)\end{array}$ & $p$ \\
\hline Normal & $153(86 \%)$ & $181(94,3 \%)$ & 0,007 \\
\hline Anomalia da glicémia em jejum & $10(5,6 \%)$ & $1(0,5 \%)$ & 0,004 \\
\hline Tolerância diminuída à glicose & $12(6,7 \%)$ & $9(4,7 \%)$ & NS \\
\hline Diabetes mellitus tipo 2 & $3(1,7 \%)$ & $1(0,5 \%)$ & NS \\
\hline
\end{tabular}

Efectivamente o actual rastreio permitiu um aumento do número de casos diagnosticados. No nosso estudo, o aumento da prevalência de DG foi de 22,8\% (4,37\% em 2009 para $5,37 \%$ em 2012) mas se considerarmos que a área de referenciação hospitalar foi alterada e consequentemente reduzida, facilmente concluímos que o referido aumento foi considerável.

Diversos autores avaliaram a prevalência de DG e obtiveram resultados semelhantes. Nwose et al determinaram o impacto no diagnóstico de DG da utilização de PTGO 75 $\mathrm{g}$ ao reavaliarem, utilizando os novos critérios, os processos clínicos de grávidas submetidas a esta prova no período compreendido entre 1999 e 2008 . Obtiveram uma taxa de diagnóstico adicional de $10,8 \%$ ao ano que se traduziu num aumento de $46 \%$ da prevalência. ${ }^{10}$ Farah et al concluíram que este rastreio se associou a um aumento da taxa de diagnóstico de $10,1 \%$ para $13,2 \%$ quando comparado com o rastreio anterior. ${ }^{11}$ Ikenoeu et al compararam os resultados perinatais de grávidas com DG, recorrendo aos novos critérios, vs grávidas com tolerância normal à glicémia, concluindo no subgrupo com apenas um valor do rastreio alterado, a intolerância à glicémia é menor mas podem estar em risco de insulinoterapia quando estão presentes antecedentes familiares de diabetes mellitus. ${ }^{12}$ Também Wery et al demonstraram um aumento da prevalência de DG para $14 \%$ ao aplicarem o rastreio universal com PTGO $75 \mathrm{~g}$ entre as 24 e as 28 semanas de gestação em 200 grávidas consecutivas. Verificaram de igual modo, um aumento do IMC pré-concepcional, maior taxa de antecedentes familiares de diabetes mellitus tipo 2 , antecedentes obstétricos de diabetes em gravidez anterior e maior peso do $\mathrm{RN}$ ao nascer quando comparado com grávidas sem DG. ${ }^{13}$

Uma das grandes falhas do actual rastreio é a ausência de diagnóstico no terceiro trimestre. Como já referido anteriormente, no estudo HAPO o período entre as 24 e as 32 semanas foi o utilizado para a implementação deste rastreio. Na nossa Instituição, o doseamento da glicémia em jejum ou eventualmente a repetição da PTGO $75 \mathrm{~g}$ no terceiro trimestre é feita em grávidas com factores de risco importantes - grávidas com IMC pré-concepcional elevado, aumento ponderal excessivo durante a gravidez, fetos com estimativas ponderais acima do percentil 90 (sobretudo à custa do perímetro abdominal) ou hidrâmnios na ecografia de rotina do terceiro trimestre. Apesar de não estar preco- nizado, os autores concluíram que uma pequena percentagem de mulheres é diagnosticada nesta altura. Efectivamente, ao analisarmos os pesos destes RN ao nascer, a média $(3317,3 \mathrm{~g})$ assemelha-se à média de peso dos $\mathrm{RN}$ no ano de 2009 (3331,9 g).

O diagnóstico mais precoce e logo em mulheres com disfunção metabólica mais ligeira permite uma actuação mais célere, prevenindo o aparecimento de complicações tipicamente associadas à DG. Numa fase inicial, a sensibilização das mulheres para esta patologia e a instituição de uma terapêutica nutricional adequada e do controlo da glicémia capilar são fundamentais. Na ausência de um bom controlo metabólico, habitualmente alcançado em 1 a 2 semanas, o recurso à terapêutica farmacológica está disponível. Actualmente na nossa Maternidade são utilizados esquemas de insulina de acção lenta ou acção rápida, antidiabéticos orais - metformina - ou a associação de ambos os grupos terapêuticos. Em qualquer um dos casos, a terapêutica nutricional mantém-se como parte integrante para o controlo metabólico adequado.

$\mathrm{Na}$ terapêutica farmacológica associada à terapêutica nutricional foi privilegiado o recurso à insulinoterapia, predominando o esquema basal com insulina humana NPH mas, por vezes, também utilizado o esquema "basal - bólus" (insulina humana NPH associada a análogo rápido); em poucos casos foi usado análogo rápido de forma isolada. Embora não seja consensual a sua utilização na gravidez, apesar de vários estudos que concluem pela sua segurança, a metformina foi utilizada quer de forma isolada ou em associação com insulinoterapia. A instituição terapêutica com este fármaco foi limitada a doentes com características associadas a insulinorresistência como um IMC pré-concepcional superior a $30 \mathrm{Kg} / \mathrm{m}^{2}$, história de Síndrome de Ovário Poliquístico ou Acantose Nigricans, assim como um maior ganho ponderal e história familiar de diabetes mellitus.

$\mathrm{Na}$ amostra, houve uma redução significativa do aumento ponderal durante a gravidez e maior utilização de terapêutica farmacológica (com significado estatístico) com uma redução significativa do peso do RN ao nascer, alcançando um dos objectivos deste rastreio. Apesar disso, a média do valor de $\mathrm{HbA} 1 \mathrm{c}$ no terceiro trimestre não sofreu variações entre os dois anos, o que vai de encontro ao que hoje em dia é amplamente aceite, que este parâmetro tem 
pouco interesse na maioria das grávidas pelo seu turnover eritrocitário que leva a muitos resultados falsos negativos. Contudo, poderá ter algum interesse em casos particulares mas no geral os autores questionam-se se este método será o melhor para avaliar o controlo metabólico da DG.

Outra das complicações associadas à DG é o parto por cesariana por suspeita de macrossomia fetal. Apesar desta indicação variar de acordo com o Centro em causa, na nossa Instituição, a estimativa ponderal (EP) superior a $4000 \mathrm{~g}$ é a utilizada. De facto, no nosso estudo, houve tendencialmente uma redução da taxa de cesarianas. As cesarianas electivas por EP acima dos $4000 \mathrm{~g}$ ou cesarianas urgentes por distócia foram reduzidas e verificou-se um aumento das cesarianas urgentes por EFNT. Provavelmente, estes fetos terão uma menor reserva de oxigénio que se traduzirá por uma menor tolerância à contractilidade uterina durante o trabalho de parto levando a um aumento de traçados de cardiotocografia suspeitos / patológicos e consequentemente maior taxa de cesarianas em trabalho de parto.

$\mathrm{Na}$ amostra, no actual rastreio a média de peso do RN ao nascer é mais baixa. Efectivamente registou-se uma redução dos RN GIG. Contudo, verificou-se uma tendência para o aumento dos RN LIG. Possivelmente, o diagnóstico mais precoce e a vigilância e tratamento mais 'intensivos' poderão explicar esta tendência. De igual modo, este facto contribuirá para o aumento da taxa de cesarianas urgentes por EFNT.

Finda a gravidez, impõe-se a realização da prova de reclassificação, importante para o futuro da mulher. Apesar da maioria das mulheres ter uma prova normal, a pequena fracção com a prova alterada permite a alteração do estilo de vida - dieta apropriada e incentivo à realização de qualquer actividade física - e a referenciação aos Cuidados de Saúde Primários.

De acordo com dados da DGS, em 2011 a taxa global de mulheres que fizeram a prova de reclassificação pós-parto foi de $68 \% .{ }^{7}$ No nosso estudo, esta taxa foi superior à estatística da população geral. $\mathrm{O}$ actual rastreio associou- -se a um aumento dos resultados normais para esta prova e a uma diminuição das anomalias da glicémia em jejum, o que provavelmente é justificado pelos critérios de diagnóstico mais apertados.

\section{CONCLUSÃO}

A DG é, sem dúvida, uma complicação da gravidez cada vez mais frequente e que tem implicações não apenas na gravidez em curso (materna e fetal) mas também no futuro da mulher e do recém-nascido.

Os autores concluíram que a introdução do actual rastreio se traduziu numa maior taxa de diagnóstico de DG apesar da redução da área de referenciação hospitalar. Houve um aumento do recurso à terapêutica farmacológica para atingir um controlo metabólico adequado, diminuição do peso médio do RN ao nascer, diminuição da taxa de RN GIG e uma tendência para o aumento de RN LIG e para a redução de cesarianas. Relativamente à prova de reclassificação pós-parto, verificou-se um aumento dos resultados normais e diminuição das anomalias da glicémia em jejum.

São necessários estudos adicionais que avaliem os resultados obstétricos e perinatais utilizando os actuais critérios de diagnóstico de DG e a eventual problemática do aumento de RN LIG.

\section{OBSERVAÇÕES}

Parte do manuscrito foi apresentado no $3^{\circ}$ Congresso Nacional de Obstetrícia e Medicina Materno-Fetal, 21 a 23 de Novembro de 2013, Porto.

\section{CONFLITOS DE INTERESSE}

Os autores declaram não ter qualquer conflito de interesse relativamente ao presente artigo.

\section{FONTES DE FINANCIAMENTO}

Os autores declaram não ter recebido qualquer subsídio relativo ao presente artigo.

\section{REFERÊNCIAS}

1. Dores J, Almeida M, Vicente L, Paiva S. Relatório de consenso sobre diabetes e gravidez. 2011. [Consultado 2013 Out 02]. Disponível em http://www.dgs.pt/?mid=5005\&cr=19703.

2. Metzger B, Lowe L, Dyer A, Trimble E, Chaovarindr U, Coustan D, et al. Hyperglycemia and adverse pregnancy outcomes. N Engl J Med. 2008;358:1991-2002.

3. Metzger B, International Association of Diabetes and Pregnancy Study Groups. International Association of Diabetes and Pregnancy Study Groups recommendations on the diagnosis and classification of hyperglycemia in pregnancy. Diabetes Care. 2010;33:676-82.

4. Reece E, Moore T. Clinical opinion - the diagnostic criteria for gestational diabetes: to change or not to change. Am J Obstetr Gynecol. 2013:255-9.

5. VanDorsten J, Dodson W, Espeland M, Guise J, Mercer B, Minkoff H, et al. National Institutes of Health Consensus Development Conference Statement - diagnosing gestational diabetes mellitus. Obstetr Gynecol. 2013;122:358-69

6. Landon M, Nicholson W. Practice Bulletin Number 137, August 2013 Clinical Management Guidelines for Obstetrician-Gynecologist: Gestational Diabetes Mellitus. Obstetr Gynecol. 2013;122:406-16.

7. Direcção Geral de Saúde. Diagnóstico e Conduta na Diabetes Gestacional. Norma número 007/2011. Lisboa: DGS; 2011.

8. Direcção Geral de Saúde. Diabetes e Gravidez. Norma número 08/ DGDG de 04/11/1998. Lisboa: DGS; 1998.

9. Saade G, American College of Obstetrics and Gynecology. Expanding the screening for diabetes in pregnancy - overmedicalization or the right thing to do? Obstetr Gynecol. 2013;122:195-7.

10. Nwose E, Richards R, Bwititi P, Butkowski E. New guidelines for diagnosis of gestational diabetes: pathology-based impact assessment. N Am J Med Sci. 2013;5:191-4.

11. Ali F, Farah A, O'Dwyer V, O'Connor C, Kennelly M, Turner M. The impact of new national guidelines on screening for gestational diabetes mellitus. Irish Med J. 2013;106:57-9.

12. Ikenoue S, Miyakoshi K, Saisho Y, Sakai K, Kasuga Y, Fukutake M, et al. Clinical impact of women with gestational diabetes mellitus by the new consensus criteria: two year experience in a single institution in Japan. End J. 2014;61:353-8.

13. Wery E, Vambergue A, Le Goueff F, Vincent D, Deruelle P. Impact des nouveaux critères de dépistage sur la prévalence du diabète gestationnel. J Gynecol Obstet Biol Reprod. 2014;43:307-13. 


\section{Diabetes Gestacional e o Impacto do Actual Rastreio}

Acta Med Port 2015:28:29-34

Publicado pela Acta Médica Portuguesa, a Revista Científica da Ordem dos Médicos

Av. Almirante Gago Coutinho, 151

1749-084 Lisboa, Portugal.

Tel: +351218428215

E-mail: submissao@actamedicaportuguesa.com

www.actamedicaportuguesa.com

ISSN:0870-399X | e-ISSN: 1646-0758

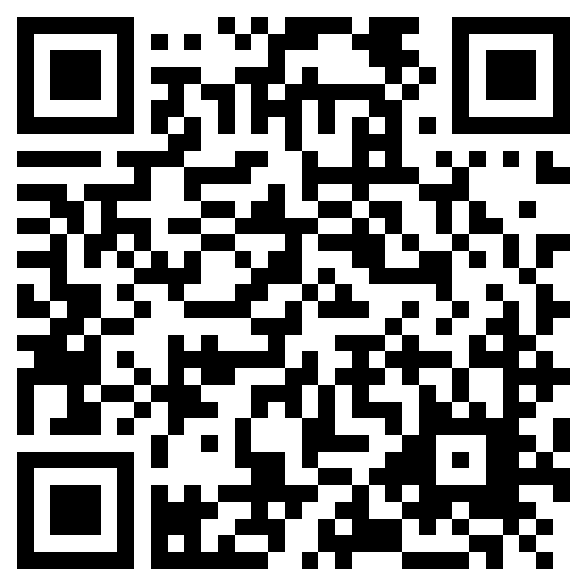

